\title{
CFD applications in offshore engineering
}

\author{
Muk Chen Ong ${ }^{1, *}$ \\ ${ }^{1}$ Department of Mechanical and Structural Engineering and Materials Science, University of Stavanger, 4036 Stavanger, Norway
}

\begin{abstract}
This paper gives a discussion on the Computational Fluid Dynamics (CFD) applications in offshore engineering. Accurate hydrodynamic quantities are essential for engineering design. Offshore structures are generally subject to high Reynolds number flows. These high Reynolds number flow conditions $\left(\operatorname{Re}>10^{6}\right)$ are difficult and expensive to achieve in an experimental setup. Therefore, it is attractive to use CFD to provide the essential hydrodynamic quantities for practical design. Verification and validation studies are important for determining the validity of the CFD prediction. A procedure of performing CFD simulation is shown. Different types of turbulence modelling are discussed. Three examples of high Reynolds number CFD simulations, covering flow around offshore structural components and waves past partially-submerged horizontal cylinders, are shown and discussed.
\end{abstract}

\section{Introduction}

The rapid developments of offshore oil and gas industry for drilling, well intervention, production and storage at sea, aquaculture industry and the exploitation of renewable energy from wind, waves and current have increased the importance of flow-structure interactions and sediment transport around offshore structures such as platform legs/columns, offshore foundations, offshore floating structures, pipelines and risers. These offshore structures are often subject to very high Reynolds number flows, especially in the open sea environment. The flow mechanisms around these structures are complex. Hence, more research of the high Reynolds number flows in the ocean is necessary in order to gain a better understanding of the flow characteristics and dynamic responses of these structures. Furthermore, high Reynolds number flows related to offshore structures are hard and expensive to measure. Therefore, an attractive alternative is to use Computational Fluid Dynamics (CFD) to obtain the essential hydrodynamic quantities needed for engineering design.

The definition of Reynolds number is $R e=U L / v$, where $U$ is the flow velocity, $L$ is the characteristic length and $v$ is the kinematic viscosity of the fluid. Generally the characteristic length of the offshore structures is large. Thus, the order of magnitude of $R e$ can range from $10^{3}$ to $10^{8}$. For cases of $R e>10^{6}$, the cost of carrying out the experiments in water basin/tank or wind tunnels is high. Moreover, scaling effect is usually an important concern for model testing of large scale structures, since it is very difficult to achieve the same flow condition as in the real life engineering application.

It is always possible to carry out field measurement. However, the data analysis for field measurement is demanding and full of uncertainties. Interpretation of the field measured data can be subjective if the amount of data is insufficient. However, when the amount of data is large, judgement of the measurement accuracy may be subjected to a vast variation.

Since there are plenty of difficulties to carry out high Reynolds number model testing and field measurement, numerical simulations should be used as an alternative to reduce the burden of experiments and field measurements. Generally detailed numerical simulations are computationally costly. It is essential to understand the flow physics before carrying out the numerical simulations, so that the complex engineering problem can be simplified without losing the essence of engineering. Verification and validation studies are important to ensure the validity of the CFD model. For example, in order to capture the boundary layer flow accurately, the near-wall mesh should be sufficiently fine. The demand of the computational resource will then be increased tremendously. Hence, engineers should have understood the flow physics well before they start performing the numerical modelling.

In this paper, applicability of turbulence models for high Reynolds will be discussed. An engineering procedure to perform CFD simulations will be discussed. Three examples of flows related to offshore engineering applications are mentioned in this paper to demonstrate the CFD applicability at high Reynolds number flow, i.e., a) flow around rectangular cylinders with different aspect ratios, (b) flow around offshore wind turbine foundations, and (c) waves past partially-submerged cylinders. 


\section{Numerical Models}

The understanding of the flow physics around the offshore structures is important for achieving accurate numerical prediction. For example, the flow condition of the boundary layer around the structure is important to determine the location of separation point, pressure distribution and skin friction distribution around the structures. This will subsequently influence the hydrodynamic quantities such drag forces, lift forces and vortex shedding frequencies. First we should identify out several important non-dimensional parameters, such as Reynolds number and Keulegan-Carpenter number, which will be the governing parameters for the variation of hydrodynamics quantities in different flow regimes. For example, for flow around circular cylinder, the timeaveraged drag coefficient is dependent on the change of Reynolds number, see Sumer and Fredsøe [1] and Ong et al. [2] for details.

Effects of turbulence on the hydrodynamic coefficients should be investigated. The geometry of the offshore structures should be also taken in consideration. The boundary layer around cylindrical-like structure will change from laminar to turbulent dependent on the corresponding Reynolds number, see Sumer and Fredsøe [1] for details. Therefore, appropriate turbulence modelling becomes essential when the boundary layers need to be resolved and computed correctly. Three dimensionality of the flow should also be considered. When the 3D effect of the flow is prominent, 3D simulations should be carried out in order to capture the correct flow physics, see Section 3 for further discussion.

Figure 1 shows a flow chart describing a procedure to perform CFD simulations including both verification and validation studies. Generally, there are four major stages: 1) Problem identification, 2) Pre-processing, 3) Solving, and 4) Post-processing. Grid and time-step convergence studies need to be carried out; and then the numerical results can be validated against available published experimental or field data.

Based on Figure 1 "Item 4: Identify flow physics", it includes the selection of turbulence modelling. For turbulent flows, complexity of flow phenomena and different range of length scales make most of the modelling approaches computationally expensive. The computational cost for resolving all the length scales in turbulence is tremendously high. Hence, a feasible approach is to create turbulence models to approximate unresolved parts in the flow. Generally, turbulence models can be categorized based on the corresponding computational cost. The more turbulence length scales are resolved, the finer the simulation resolution is, and consequently the higher the computational cost will be. However, if most of the turbulent length scales are not resolved (i.e. low computational cost), the poor numerical accuracy is expected to be obtained. Moreover, the Navier-Stokes equations contain nonlinear convection and pressure gradient terms; and these nonlinear terms have to be solved numerically with appropriate boundary conditions.

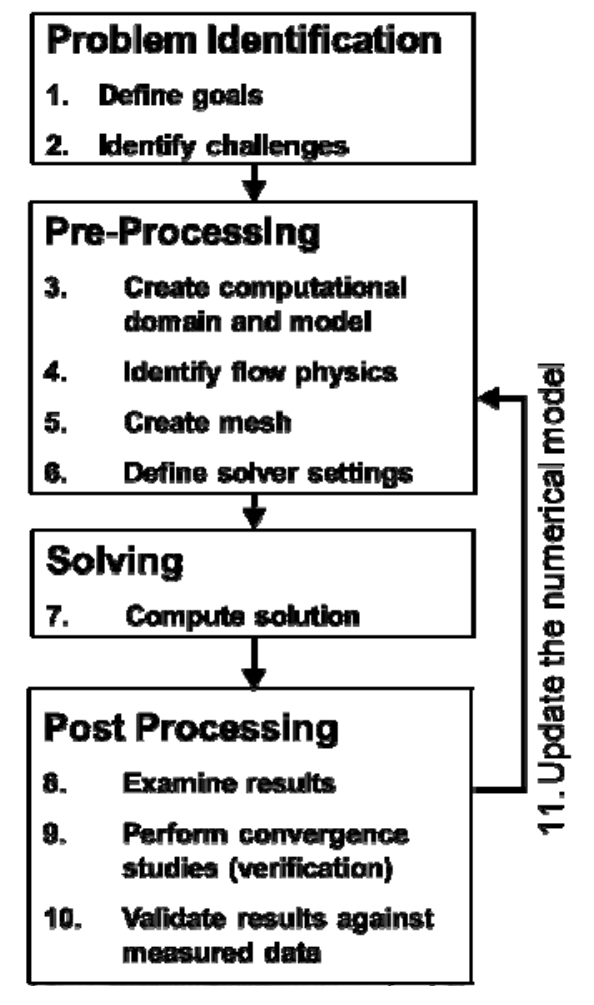

Fig. 1. A procedure to perform CFD simulations including both verification and validation studies.

In operating conditions, offshore structures are often subject to high Reynolds number flow conditions. At high Reynolds numbers, flows are turbulent. Turbulent flows are highly irregular; however, they are governed by the exact Navier-Stokes equations. Efficient numerical algorithms solving the Navier-Stokes equations and the turbulence models for high Reynolds number flows are still considered as a major challenge although many numerical methods (e.g. SIMPLE-like class of algorithms and projection methods) have been developed. Furthermore, the appropriateness of turbulence models for the targeted engineering applications should be considered carefully. The computer and cost efficiency of the computational method is also important, especially for engineering applications.

Direct Numerical Simulation (DNS) resolves the whole range of spatial and temporal scales of the turbulence in the flow. A DNS simulation requires a number of mesh points more than $R^{2.25}$ [3]. It makes the computational cost extremely high for flow around complex geometries at high Reynolds number. Hence, hydrodynamic analyses based DNS simulations are currently not feasible for offshore industry.

Reynolds-averaged Navier-Stokes (RANS) model is the most commonly-used numerical approach for offshore engineering applications. All of the unsteadiness in the Navier-Stokes equations is taken as part of turbulence and is averaged out in the Reynolds- 
averaged approach. In the averaging process, the nonlinearity of the Navier-Stokes equations gives the fluctuation terms which needs to be modelled. Turbulence models, such as k- $\varepsilon$ model, $\mathrm{k}-\omega$ model, $\mathrm{k}-\omega$ SST model and Spalart-Allmaras model, can be used as a turbulence closure. More details can be referred to Ferziger and Perić [4].

Large-eddy simulation (LES) is a numerical approach, in which large-scale motions are explicitly computed and small-scale eddies are modeled with subgrid scale models. LES is three-dimensional, time dependent and less expensive than DNS for the same flow. LES is the preferred method when the Reynolds number is too high or the geometry is too complex to allow the application of DNS [4].

Detached Eddy Simulation (DES) is a hybrid RANSLES model, in which the model switches from RANS to LES (subsgrid scale formulation) in regions where the mesh resolution is sufficiently fine for LES. Therefore, the grid resolution for DES is not as high as pure LES, and subsequently the computational cost is reduced.

Three examples related to offshore engineering applications at high Reynolds number flow will be shown in Section 3. RANS, LES and DES are employed in these simulations to demonstrate the applicability of CFD approach.

\section{Examples}

\subsection{Flow around rectangular cylinders with different aspect ratios}

Many marine and offshore structures have structural components with square or rectangular cross-sections, such as columns of semi-submersibles and tension-leg platforms, as well as heave damping plates of truss spar platforms. Two-dimensional flow normal to the longer edge of rectangles with different aspect ratios $(R=B / H)$, i.e. from a square to a thin plate, has been investigated numerically in Tian et al. [5] at $R e=21400$. Here $B$ is the breadth along the direction of flow and $H$ is the height of the cross-section. The objective of the study is to evaluate the validity of $2 \mathrm{D}$ unsteady RANS (URANS) simulation with k- $\omega$ SST model for flow around rectangular cylinders. Figure 2 shows the comparison of the mean drag coefficient $\overline{C_{D}}$ between 2D URANS results reported by Tian et al. [5] and the published 2D and $3 \mathrm{D}$ numerical results as well as experimental results [6-12]. The drag foces acting on the cylinders with high aspect ratios (i.e. $R=1,0.8$ and 0.6) are well predicted by the numerical simulations; however, the drag forces are over-predicted for low aspect ratios (i.e. $\mathrm{R}=0.4,0.2$, 0.1 and 0.05$)$.

Tian et al. [13] performed both 2D URANS and 3D LES simulations to investigate the 3D effects of the flow normal to a flat plate with an infinite spanwise length at $R e=1.5 \times 10^{5}$. The ratio of the plate thickness to the plate height is 0.02 . The hydrodynamic results (such as timeaveraged drag coefficient, Strouhal number and mean recirculation length) were compared with the published experimental data reported by Fage and Johansen [8] and Leder [14]. The 3D LES results are in good agreement with the published experimental data; however, the 2D URANS shows a poor comparison with experimental data. This feature is similar to the conclusion obtained by Tian et al [5] for rectangular cylinders with small aspect ratio. Figure 3 shows the instantaneous vortical structures in the wake reported by Tian et al. [13]. A highly $3 \mathrm{D}$ and chaotic wake flow is observed (especially the secondary flow in the spanwise direction) although the plate with infinite spanwise length is nominally $2 \mathrm{D}$. Figure 4 shows the streamlines of the mean velocity field obtained in the 2D and 3D simulations (taken from Tian et al. [13]). It shows that the recirculation length in the $2 \mathrm{D}$ simulation is approximately two times larger than that in the $3 \mathrm{D}$ simulation. The $3 \mathrm{D}$ flow effects are prominent for high Reynolds number flow normal to a flat plate. Tian et al. [13] reported that the reliability of $2 \mathrm{D}$ simulations for the nominally $2 \mathrm{D}$ problem is questionable and more attention should be made to investigate the $3 \mathrm{D}$ effects.

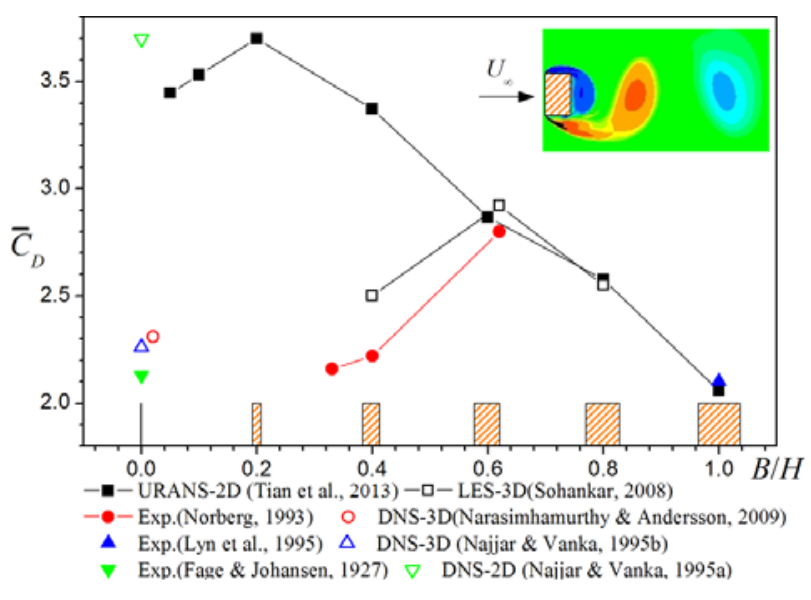

Fig. 2. Comparison of mean drag coefficients between 2D URANS results reported by Tian et al. [5] and published experimental and numerical results [6-12] for flow around rectangular cylinders with different aspect ratios.

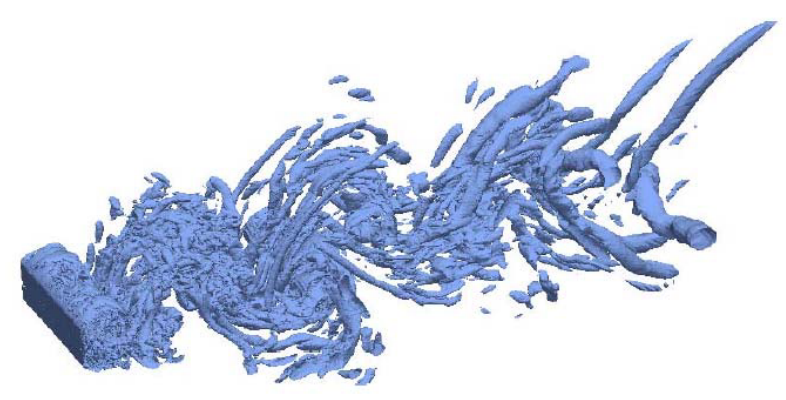

Fig. 3. Instantaneous vortical structures in the wake of flow normal to a thin flat plate (taken from Tian et al. [13]). 

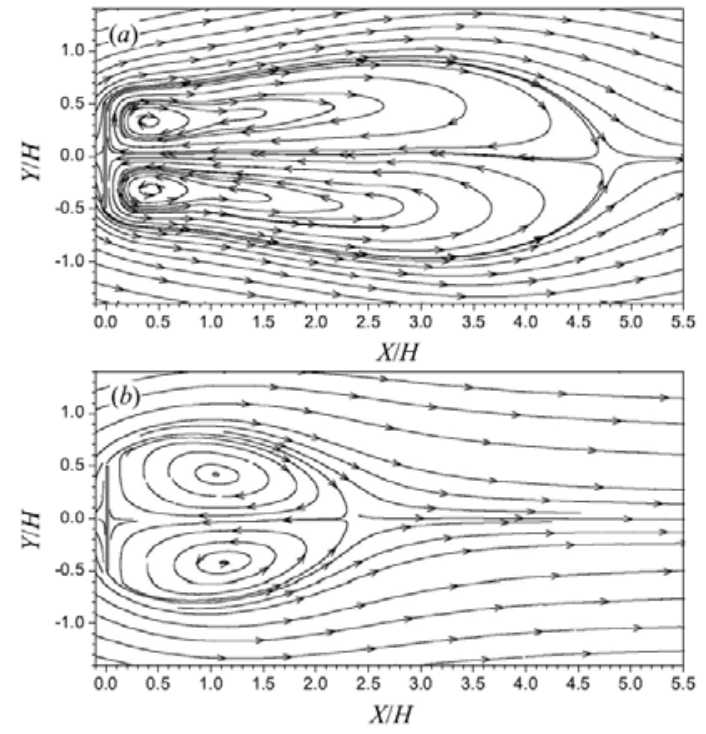

Fig. 4. Streamlines of the mean velocity field obtained in the 2D (top) and 3D (bottom) simulations (taken from Tian et al. [13]).

\subsection{Seabed boundary layer flow around offshore wind turbine foundations}

Ong et al. [15] investigated the formation of horseshoe vortex and flow structures around monopile and gravitybased offshore wind turbine foundations in order to provide an assessment of potential scour for engineering design. Three-dimensional CFD simulations were performed using Spalart-Allmaras Delayed Detached Eddy Simulation (SADDES) at $R e=4 \times 10^{6}$ based on the free stream velocity and the diameter of the monopile foundation. Grid and time-step convergence studies (verification study) were performed. Figure 5 shows 3D views of the mesh on the surface of the monopile and hexagonal gravity-based foundations (Ong et al. [15]).

Figures 6(a) and (b) show 3D views of instantaneous vortical structures around the monopile and hexagonal gravity-based foundation, respectively (Ong et al. [15]). A distinct horseshoe vortex is found in front of the monopile foundation. Two small horseshoe vortices are found in front of the hexagonal gravity-based foundation, i.e. one is on the top of the bottom slab (" 1 " marked in Figure 6(b)) and one is near the seabed in front of the bottom slab ("2" marked in Figure 6(b)). This flow feature reduces the strength of the horseshoe vortex on the seabed for the hexagonal gravity-based foundation.

The size of the horseshoe vortex can be determined from the time-averaged bed shear stress $\tau$ along the centreline from the inlet to the location of the foundation toe. Figures 7 (a) and (b) shows the normalized timeaveraged bed shear stress $\tau / \tau_{\infty}$ along the centreline for the monopile and hexagonal gravity-based foundations, respectively, where $\tau_{\infty}$ is the time-averaged undisturbed bed shear stress. Ong et al. [15] reported that the hexagonal gravity-based foundation shows a reduction of the horseshoe vortex size as compared with that for the monopile foundation under the same flow condition.

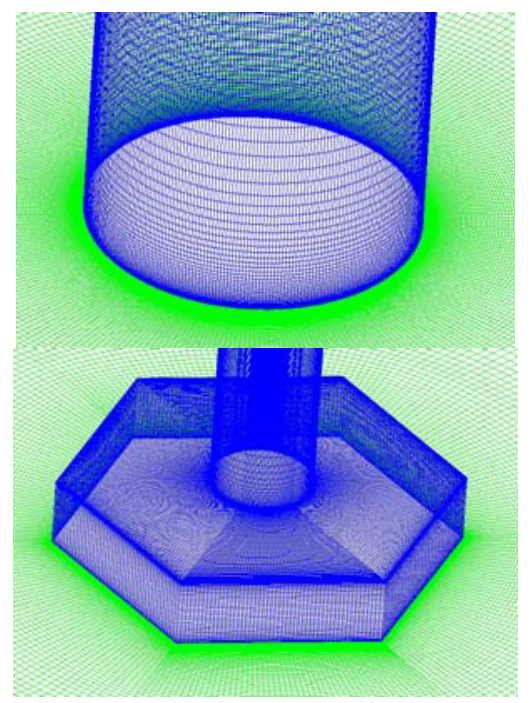

Fig. 5. 3D views of the mesh on the surface of the monopile and hexagonal gravity-based foundations (taken from Ong et al. [15]).

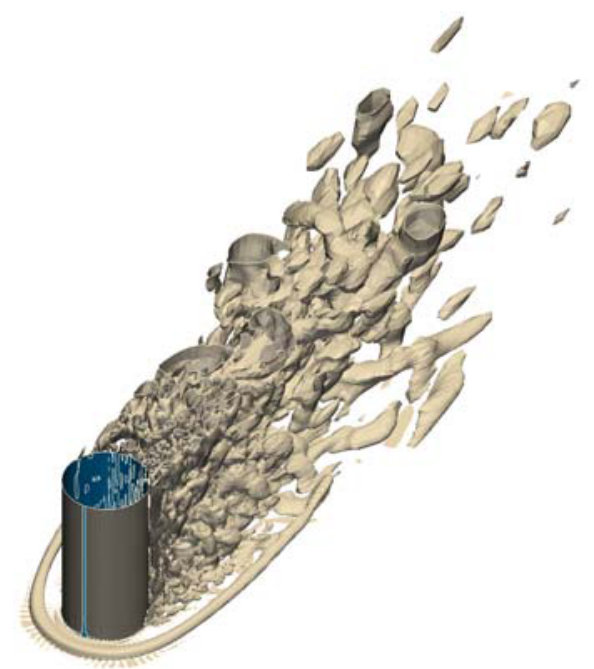

(a) Monopile foundation

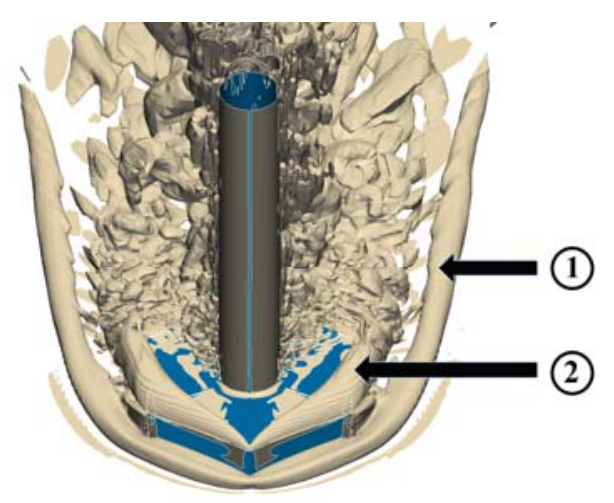

(b) Hexagonal gravity-based foundation

Fig. 6. 3D views of instantaneous vortical structures around the monopile (top) and hexagonal gravity-based (bottom) foundations (taken from Ong et al. [15]). 


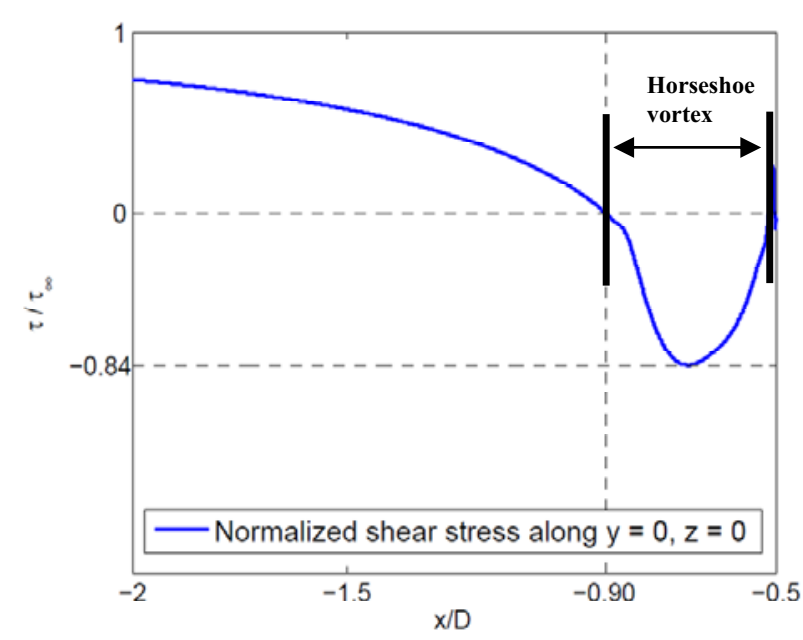

(a) Monopile foundation

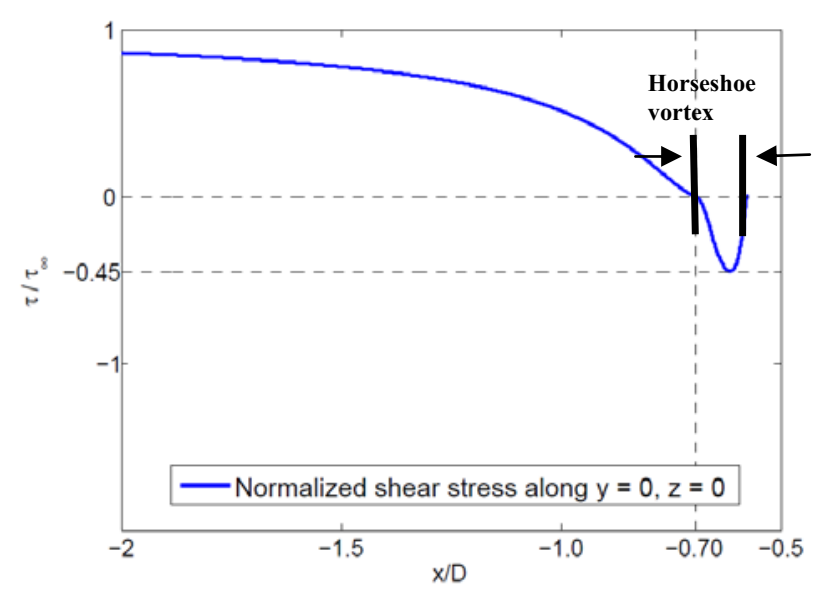

(b) Hexagonal gravity-based foundation

Fig. 7. The normalized time-averaged bed shear stress along the centreline for the monopile (top) and hexagonal gravitybased (bottom) foundations (taken from Ong et al. [15]).

\subsection{Free Surface Waves past Partially Submerged Horizontal Cylinders}

Partially-submerged bluff bodies are often found in offshore structures, e.g. wave energy converter and fish cages. Circular cylinders are usually one of the important components in these structures. Bihs and Ong [16] performed 2D CFD simulations to investigate the flows past partially-submerged circular cylinders in free surface waves. The simulations were carried out by solving the URANS equations with the $\mathrm{k}-\omega$ model. The level set method was employed to model the free-surface waves. Vertical wave forces on the partially-submerged cylinders have been calculated numerically and compared with experimental data reported by Dixon et al. [17] under regular-wave conditions. A Grid convergence study was performed to obtain sufficient mesh resolution. The investigated Keulegan-Carpenter number ranged from 1.26 to 3.14 . Figure 8 shows the normalized vertical hydrodynamic force $\left(F v^{\prime}\right)$ versus the non-dimensional time $\left(t^{\prime}\right)$ over one wave period. The wave amplitude is half of the cylinder diameter $(D)$ and the wave length is $15.62 D$. Bihs and Ong [16] reported that the numerical prediction of the vertical hydrodynamic forces is in good agreement with the experimental data reported by Dixon et al. [17]. Figure 9 shows the free-surface elevations around the cylinders. Both wave run-up and overtopping actions are observed clearly in the simulation.

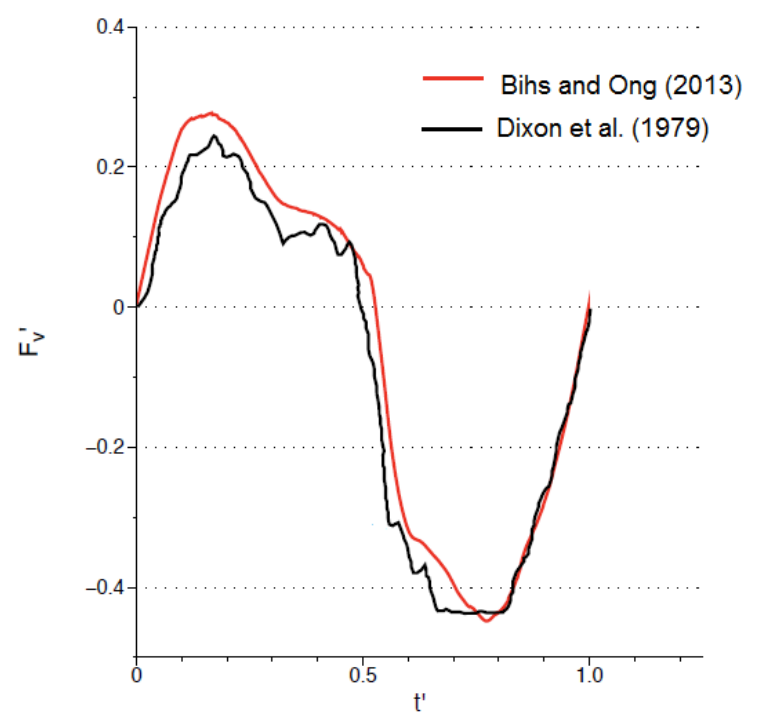

Fig. 8. The normalized vertical hydrodynamic force versus the non-dimensional time over one wave period (taken from Bihs and Ong [16]).

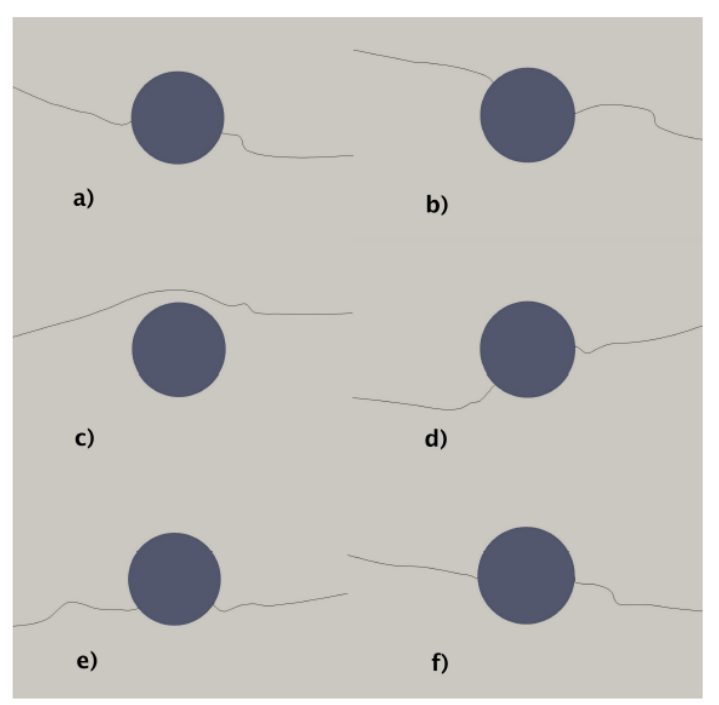

Fig. 9. Time history of free surface elevation over a wave period for (a) $\mathrm{t}^{\prime}=0.0$, (b) $\mathrm{t}^{\prime}=0.12$, (c) $\mathrm{t}^{\prime}=0.36$, (d) $\mathrm{t}^{\prime}=0.6$, (e) $t^{\prime}=0.73$, (f) $t^{\prime}=1.0$

\section{Conclusions}

Offshore structures are generally subject to high Reynolds number flows. These high Reynolds number flow conditions $\quad\left(\operatorname{Re}>10^{6}\right)$ are difficult and expensive to achieve in an experimental setup. An alternative method is to employ CFD modelling to obtain the 
essential hydrodynamic coefficients for practical engineering design.

A procedure of performing CFD simulations has been shown with the emphasis on verification and validation studies. Grid and time convergence studies (verification) and comparisons with available experimental data (validation) are important to ensure the validity of the CFD prediction. Due to the demand of high mesh resolution, DNS simulations are not feasible for applications in offshore industry. RANS and URANS simulations are commonly used to predict high Reynolds number engineering flows due to lower computational cost and reasonable accuracy of hydrodynamic prediction. DES and LES simulations are employed when detailed flow structures are essential for practical design.

Three examples, i.e. i) flow around rectangular cylinders with different aspect ratios, ii) seabed boundary layer flow around offshore wind turbine foundations, iii) free surface waves past partiallysubmerged horizontal circular cylinders, have been shown and discussed to demonstrate the applicability of CFD simulations for offshore engineering design.

\section{References}

1. B.M. Sumer, J. Fredsøe, Hydrodynamics around Cylindrical Structures: Advanced series on ocean engineering, 12 (1997).

2. M.C. Ong, T. Utnes, L.E. Holmedal, D. Myrhaug, B. Pettersen, Marine Structures, 22, 142-152 (2009).

3. S.B. Pope, Turbulence Flows (2000).

4. J.H. Ferziger, M. Perić, Computational Methods for Fluid Dynamics (2001).

5. X. Tian, M.C. Ong, J. Yang, D. Myrhaug, Ocean Engineering, 58, 208-216 (2013).

6. C. Norberg, Journal of Wind Engineering and Industrial Aerodynamics, 49 (1-3), 187-196 (1993).

7. D.A. Lyn, S. Einav, W. Rodi, J.H Park, Journal of Fluid Mechanics, 304(1), 285-319 (1995).

8. A. Fage, F.C. Johansen, Proceedings of the Royal Society of London. Serie A, 116, 170-197 (1927).

9. A. Sohankar, Journal of Wind Engineering and Industrial Aerodynamics, 96, 640-655 (2008).

10. V.D. Narasimhamurthy, H.I. Andersson, B. Pettersen, International Journal of Heat and Fluid Flow, 30, 1037-1043 (2009).

11. F.M. Najjar, S.P. Vanka, Physics of Fluids, 7, 25162518 (1995).

12. F.M. Najjar, S.P. Vanka, International Journal of Numerical Methods in Fluids, 21, 525-547 (1995).

13. X. Tian, M.C. Ong, J. Yang, D. Myrhaug, G. Chen, OMAE Conference, OMAE2012-83730 (2012).

14. A. Leder, Physics of Fluids A: Fluid Dynamics, 3, 1445 (1991).

15. M.C. Ong, E. Trygsland, D. Myrhaug, OMAE Conference, OMAE2016-54643 (2016).

16. H. Bihs, M.C. Ong. OMAE Conference, OMAE2013-10529 (2013).
17. A.G. Dixon, C.A. Greated, S.H. Salter, Journal of the Waterway, Port, Coastal and Ocean Division, 105 (4), 421-438 (1979). 